\title{
CHRONIC LEG ULCERS
}

\section{Aetiology}

\author{
By S. T. ANning, T.D., M.A., M.D.(Cantab.), M.R.C.P. \\ Assistant Physician, Dermatological Department, The General Infirmary at Leeds
}

'The Legges ... . when they are offended or wounded, are very perillous, because unto them runneth a great quantity of Humors.'

THOS. VICARY (1495-r 56r).

Leg ulcers are common and there are many varieties. Although those associated with venous defects are the most common and will be dealt with more fully here, they result, not from a single cause, but from the interaction of several complex factors. Only by an attempt to analyse these can we hope to establish a rational system of treatment.

In the aetiology of ulceration of the leg two factors are of particular importance-trauma and certain peculiarities in the circulation of the blood in the lower limbs.

The legs are exposed to injury of many kindskicks from the other foot or that of other people, grazes, knocks, sprains and insect bites. These and other causes may result in damage to the skin or venous thrombosis or both. Women are less exposed to violence, but their legs are not so well protected as those of men.

Traumatic ulcers may appear on the legs of healthy individuals after the skin has been broken. Delay in healing may result from constant movement of the part, secondary infection or, more important, because of certain circulatory factors which must be considered.

\section{The Circulation in the Lower Limb}

In discussing the circulation of the lower limb in relation to leg ulcers it is necessary to think in terms of the blood flow of the limb as a whole and not merely that in certain veins. For example, it is being increasingly appreciated that, even with 'venous ulcers,' the arterial blood flow is of importance and, associated with this, the opening up of arterio-venous shunts.

The adoption of the upright posture by mankind imposed a strain on the mechanism which maintains the circulation of blood from the lower limb, because the blood returning to the heart required to be pumped twice the height formerly necessary. It is not without significance that man is unique among mammals in suffering from leg ulcers.

Although the weight of the column of blood the arterial part of the vascular system of the lower limb is balanced by that in the venous ( in a U-tube) the vis a tergo driving the venofs blood upward towards the heart is only that of the capillary less the venous pressure. A relay pumping system is required and is providect by the calf muscles and the valves in the deep velin? On contraction of the muscles the blooe of squeezed upward in the deep veins and on reloxp tion the venous valves prevent the fall of blood downwards. Tonic contraction of the muscles grs in standing at attention, in no way assists venous return. The blood flows from the suplert ficial venous trunks (internal and external saphe nous) into the deep trunks (popliteal and femoraf) and, as Cockett (1955) has shown, blood from the region of the ankle drains into the post-tibial through perforating veins. The flow of blood from the superficial venous system into the deêp indicates that in at least one phase of action of the muscle pump the pressure in the dee veins must be lower than that in the superficial. This can only be during diastole of the pump. $\frac{0}{3}$

This concept of the haemodynamics in the lower limb is confirmed by measurement of the pressure in the superficial veins, the posterior

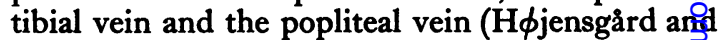
Stürup, 1952). In a normal person standing stîfl the pressure in these veins is the same and $P S$ almost equal to the calculated haemostatic pres sure (i.e. the weight of a column of blood from the point of measurement to the right side of the heart) which depends purely on the height of the individual. During walking there is a rapid f f in the pressure in the superficial veins and in the posterior tibial veins but not in the popliteal vein. The pressure in the latter could not be expected 
to fall during walking as the measurement is taken above the muscle pump. Nevertheless, it is necessary to repeat that the pressure in the popliteal and femoral must be less than that in the external and internal saphenous veins, at least during some phase in the process of walking, or blood would not flow from the superficial veins into the deep.

\section{Varieties of Leg Ulcer}

As already mentioned, gravitational or venous ulcers are the commonest and other varieties will only be mentioned briefly here.

\section{Classification of Leg Ulcers}

r. Circulatory:

(a) Arterial-

Arteriosclerotic ulcer

Hypertensive ulcer

(b) Arteriolar-

Polyarteritis nodosa (rare)

Erythrocyanosis with ulcers

Allergic:

Erythema induratum (tuberculous)

Tertiary gumma (syphilitic)

Nodular vasculitis (allergen unknown)

(c) Venous-

Post-thrombotic ulcer

Varicose ulcer

(d) Congenital arterio-venous anastomosis with ulceration

2. Associated with other diseases:

Rheumatoid arthritis

Ulcerative colitis

Blood diseases

3. Neoplastic:

Primary epithelioma

Leukaemia (breakdown of deposit in the skin)

\section{Arterial Ulcers}

Arteriosclerosis affects large and medium sized vessels. Although not a cause of hypertension it is more frequent in subjects with raised than with normal pressures and it may be associated with diabetes mellitus. Slow occlusion of the arteries by atheroma and the gradual deposition of thrombus may occur or there may be sudden impairment of the blood flow from blockage by thrombus. In the lower extremity gangrene of the toes is the more common result but patches of gangrene, sometimes precipitated by slight trauma or mild infection of the skin, may appear over the feet or legs and produce deep, sloughing ulcers with exposure of the tendons.

Hypertensive ulcers, first described by Martorell (1945), are the result of changes in the smallest arteries and arterioles where the pressure gradient in the circulation is greatest. Hypertrophy and degeneration affecting the muscle of the media and the internal elastic lamina occur with a reduction in the size of the lumen. Thrombosis, perhaps provoked by mild trauma, occurring in the arterioles of the skin which are located chiefly in the deeper portion of the dermis, results in a necrotic area and a shallow ulcer very different from the deep ulceration which follows the occlusion of a large arteriosclerotic vessel. These hypertensive ulcers are rare but more common in women and are usually seen on the anterolateral aspect of the leg at about the junction of the middle and lower thirds (Martorell, 1953; Anning, 1954).

\section{Arteriolar Causes of Ulceration}

In addition to hypertension other arteriolar changes may lead to ulceration. Erythrocyanosis, which occurs with a chilblain type of circulation (perniosis), results from reduced arteriolar flow. It may be seen over the calves and the lower part of the legs and may be a constitutional defect, often familial and usually present in adolescent girls and young women, associated perhaps with at congenital narrowing of arterioles together with spasm. On the other hand it may be acquired, as? after poliomyelitis. Especially during the winter chilblains with superficial erosions may appear on the calves of those with erythrocyanosis.

Allergic reactions may occur in the sub-dermalo arterioles and may be sufficiently severe to lead to endarteritis, blockage of the vessels and necrosis. Clinically these reactions are manifest first as sub-dermal nodules, usually in the calves, with discoloration of the overlying skin and later with extension superficially of the inflammatory, necrotic process producing ' punched-out,' deep ulcers. After healing depressed scars may remain. These conditions may be associated with erythrocyanosis and may be provoked by a variety of allergens such as the tubercle bacillus many years after the primary infection (erythema induratum), the spirochaete of syphilis (in the tertiary phase), and various drugs, but the allergen may not be determined and the condition may be called nodular vasculitis. It may be that the slowness of the arteriolar flow in the calves of these individuals determines the site of the allergic reaction but it must be noted that syphilitic gummata, though provoked by a similar allergic reaction, are not associated with erythrocyanosis and occur more commonly near the knee or in other parts.

Congenital Arterio-Venous Anastomoses with Ulceration

Congenital arterio-venous anastomoses may 
occur in the lower limb or elsewhere. The affected limb is often longer than the other, varicose veins may appear at an early age and cutaneous haemangiomata may be present. Murmurs are usually audible on auscultation. Such congenital anastomoses are rare but ulceration of a limb so affected is common (Coursley et al., 1956).

\section{Ulcers Associated with Other Diseases}

Little is known about the cause of ulceration of the legs associated with arthritis. If the knee and ankle joints are affected the efficiency of the leg muscle pump is impaired and the gravitational type of ulcer may result. But ulceration does occur with rheumatoid arthritis which cannot at present be explained. The same is true of the extensive ulceration of the legs, often phagedaenic, which is found in about $I$ per cent. of patients with ulcerative colitis even without venous thrombosis which may well occur in patients with such a chronic disease requiring long periods of recumbency and associated with dehydration.

Certain blood diseases (in the absence of venous thrombosis which, for example, is liable to occur with polycythaemia vera) may be associated with leg ulcers: sickle-cell anaemia, Mediterranean anaemia, haemolytic anaemia with spherocytosis, and congenital haemolytic icterus are examples. In addition Banti's syndrome and that described by Felty (chronic rheumatoid arthritis, splenomegaly and leucopenia) must be included in this group. The one common factor appears to be hypersplenism and if splenectomy is carried out the ulcers heal. Furthermore, mere enlargement of the spleen does not cause ulceration for it is not seen associated with leukaemia (unless leukaemic infiltration in the skin of the leg breaks down) or in pernicious anaemia (unless venous thrombosis occurs).

\section{Neoplastic Ulcers of the Legs}

After many years an extremely small proportion of leg ulcers becomes neoplastic but here we are concerned with cancerous ulcers arising in normal skin. Of 1,500 patients with leg ulcers there were two with epitheliomatous ulcers of this type. As already mentioned leukaemic deposits in the leg may ulcerate.

\section{Venous Ulcers}

The essential cause of venous ulcers is failure of the leg muscle pump which results in chronic oedema of the leg, pigmentation, indurated plaques and ulceration. All these complications are more common over the lower third of the leg but not over the foot as the support of a shoe prevents the appearance of oedema there. A considerable proportion of venous ulcers appear on the lowed third of the medial aspect of the leg and this areat has been shown by Cockett (1955) to be drained directly into the deep venous system by veins? perforating the deep fascia. This confirms and elaborates Hilton's findings (186r). Ulceration may be preceded by dilatation of the tiny super ficial veins in the skin in the proximity of the malleoli. This is sometimes referred to as the ' ankle blow-out syndrome' (Cockett and Joneso 1953) and is thought to be the result of valvulap incompetence and increased pressure in the ankl 1 perforating veins. There is also evidence tha $\$$ peculiarities of the arterial supply play a part in making this site more prone to ulceration.

\section{Causes of Failure of the Muscle Pump}

Failure of the leg muscle pump may result frong a disorder of function or from organic disease $\Xi$ Thus, lack of muscular contraction, as in thosét who stand still for long periods, puts the pump ouf of action. Usually only transitory oedema results but, as already mentioned, if the knee and ankles joints are seriously affected by arthritis, chroni\& oedema and gravitational ulceration may occuro Usually, however, lack of muscular contraction not the primary cause of ulceration but ofly augments other causes of failure of the pump whers are often the result of organic disease.

\section{Deep Venous Thrombosis}

It is generally accepted that thrombosis in ilio-femoral and/or the posterior tibial veins is important, and probably the most commo cause of failure of the leg muscle pump producings chronic oedema of the leg and other complications such as ulceration.

Deep venous thrombosis does not necessarilyo completely block the affected veins but usually seriously embarrasses the return flow of blood from the lower limb as 90 per cent. of the flow normally passes through these deep veins. Even though re-canalization occurs, the valves are damaged or destroyed and permanent incompetence of the muscle pump remains.

There has been some difference of opinion as to the frequency of deep thrombosis as a cause of legi ulcers. The incidence of post-thrombotic ulcerş was found by Bauer (1946) to be 80 to 90 per cent 0 and by Anning (1954) in a series of 993 venous ulcers to be about 75 per cent. Boyd et al. (1952) investigated 308 patients with chronic leg ulcers. and, excluding 46 with arteriosclerotic ulcers $N$ 70.5 per cent. were post-thrombotic and the remainder varicose. Many authors (e.g. Dod\% and Cockett, 1956) believe the incidence of post $-\omega$ thrombotic ulcers to be lower but the figurese quoted are from quite large series and from varyinge 
types of clinic. However, it is generally agreed that venous thrombosis is an important cause of leg ulcers and this is confirmed by the incidence of ulceration as a sequel of such thrombosis. Bauer (1942) found that of 100 individuals examined more than ten years after deep thrombosis all had swelling of the legs and four-fifths had ulcers. Of 420 patients who had thrombosis previously and were examined by Servelle (r950), 2 I per cent. had leg ulcers. H $\phi$ jensgård (1952) examined I9 limbs affected with deep thrombosis 6 to 31 years previously and found that 28 per cent. were ulcerated.

\section{Varicose Veins}

Are varicose veins a cause of leg ulcers ? The importance or otherwise of superficial varicose veins in causing chronic venous insufficiency and ulceration is still a matter for dispute. Certain it is that many individuals with severe varices do not suffer from either. Furthermore, patients with chronic venous insufficiency and ulceration are seen not uncommonly with no varicose veins visible or palpable.

There is much we have yet to learn about varicose veins. For example, we know little of the place of arterio-venous anastomoses, endocrines and veno-dilator substances in their aetiology. The haemodynamics of varicose veins are still not well understood.

In the aetiology two factors appear to be of primary importance and it would seem that unless both are present varicosities do not appear. These factors are venous hypertension and an inherent weakness of the vein walls.

Venous hypertension, as we have noted, occurs in the lower limbs during periods of standing and is present when there is increased intra-abdominal pressure whether from pregnancy (or other abdominal swelling), coughing or straining. Deep venous thrombosis is an important cause of hypertension in the superficial veins which are required to carry a greatly increased quantity of blood, and Brewer (1950) produced evidence that arterio-venous shunts are opened up after such thrombosis. Such shunts would clearly cause a considerable rise in intravenous pressure. The work of Piulachs and Vidal-Barraquer (1953) and Vogler and Gollman (1955) suggests that arteriovenous shunts are of great importance in the aetiology of varicose veins.

The tendency to develop varicose veins is inherited as a dominant character and is probably related to an inherent weakness of the walls of veins rather than of their valves. Unless this inherited defect is present it seems doubtful whether varicosity will develop even in the presence of venous hypertension (though the defect must occasionally arise as a mutation in the absence of any familial tendency to varicosity). Failure of an individual to develop varicosity of the superficial veins after severe deep thrombosis may mean that the defect is not present in his family or that he is one of the 50 per cent. of siblings in an affected family who do not inherit the dominant defect.

In the presence of the inherited defect venous hypertension is more likely to cause varicosity of the superficial veins because they are not so well supported by deep fascia and muscle. May, however, there be occasions in which the deep veins become dilated and their valves defective? The condition was described by Verneuil (1855) and by Gay (1868) and has been demonstrated phlebographically by Moore (I95I). Bauer (1948) described 'idiopathic or non-thrombotic femoral incompetence.' We have here a reasonable explanation of the difference between those who have gross superficial varicose veins with no complications and those whose superficial veins are no worse but in whom oedema, pigmentation and ulceration develop. In the latter group not only are the superficial veins involved but the deep also, as a result of which the leg muscle pump is affected and chronic venous insufficiency results. It is to this group that the term ' varicose ulcer' may reasonably be applied even though it is not the superficial varicosities which are responsible.

Do then superficial varicose veins play no part in the cause of ulceration? As long as the leg muscle pump is functioning efficiently the venous return is adequate and no complications arise in spite of a reflux down the varicose saphenous veins. The pump has sufficient reserve. If, however, the pump's action is reduced, as by long periods of standing, or if the circulatory requirement of the limb is increased as by severe anaemia, inflammation or injury, the signs of chronic venous insufficiency may appear, including ulceration. This does not occur frequently.

The diagnosis of varicose ulcer is based on circumstantial evidence unless proved phlebographically. It can reasonably be made when there are signs of chronic venous insufficiency in a patient with no history of venous thrombosis, with a family history of varicose veins and when superficial varicose veins are present with a thrill palpable on coughing, the patient being erect.

Although in any series of patients with venous ulcers some will give a definite history of previous deep thrombosis when a careful history is taken and some will be diagnosed as suffering from varicose ulcers, there remains a group with no history of thrombosis and in which the diagnosis of varicose ulcer is not justifiable. Some patients 
in this group have no varicose veins and in many it may be difficult or impossible to elicit a reliable history. It seems best to classify these as venous ulcers of doubtful origin though many are probably post-thrombotic. In a series of 1,511 venous leg ulcers 73.19 per cent. were post-thrombotic, 12.5 per cent. were varicose, and I4.29 per cent. were venous ulcers of doubtful origin.

\section{The Results of Failure of the Muscle Pump}

The results of failure of the leg muscle pump can only be dealt with here briefly but it must be appreciated that the primary result is venous, and hence capillary, hypertension. This causes oedema of an entirely different type from that associated with cardiac failure, renal disease (acute and chronic) and lymphatic obstruction, in none of which is the capillary pressure raised to nearly the same degree. In deep venous disease the pressure in the veins of the foot during exercise is 50 to $I 10 \mathrm{~mm}$. mercury ( 70 to $150 \mathrm{~cm}$. water) instead of the normal 0 to $30 \mathrm{~mm}$. mercury (o to $40 \mathrm{~cm}$. water) (Walker, $195^{\circ}$ ), and the capillary pressure is even higher. As a result there is considerable capillary damage with loss of fluid containing protein and red blood corpuscles. The tissue fluid becomes organized and there is induration of the affected area. Probably a low grade infection is present and there is some blockage of the superficial lymphatics. Such an indurated area may break down 'spontaneously' or be injured, an ulcer resulting. Sometimes atrophy of the skin occurs especially over the dilated small veins seen with the 'ankle blow-out syndrome.' These atrophic patches readily break down to form small ulcers.

\section{Miscellaneous Factors in the Aetiology of Leg Ulcers}

The influence of heredity in the aetiology of leg ulceration is a large subject and has been discusse at length elsewhere (Anning, 1954). Not ony may varicose veins (or rather the tendency to develop them) be inherited, venous thrombosis also appears to be a familial disease. It is wonder then that venous ulcers may afflict several members of a family.

Obesity has been considered important in the aetiology of leg ulcers but in a thousand patients $4 \mathrm{I}$ per cent. of the $69 \mathrm{I}$ women and 31 per cerir. of the 309 men weighed less than $70 \mathrm{~kg}$. (I $54 \mathrm{llg}$ ) (Anning, 1954). The patients with post-thrombote were heavier than those with varicose ulces. In brief, leg ulcers, and particularly varicose and arterial ulcers, occur in many patients whose weight is normal. Nevertheless, when a patienteds obese reduction of weight assists healing of the ulcer.

The association of hypertension with ulceration of the venous type is a curious finding which has not been fully explained (Anning, 1954). Muen more work requires to be carried out on this subject.

From the time of Hippocrates the importanoe of occupation in the aetiology of leg ulcers thas been appreciated and it was noted by Ramaizini in his book on 'The Diseases of Tradesmper' (I 705). It is sufficient to repeat here that ueess are more common in those who stand for toog periods while at work.

\section{Summary}

In the aetiology of leg ulcers many factors afe at work. The majority of ulcers are of the 'venous' type and the essential cause is failuge of the leg muscle pump. Superficial varicoge veins play only a minor role. The importange of heredity, hypertension and occupation must not be forgotten.

\section{HOW TO GET THERE}

An Address Book for the Medical profession, showing how to reach the various Colleges, Societies, Institutes and Hospitals in or near London

New (Fourth) Edition: 1954

Price 2s. 6d. (2s. 10d., post free)

Published by the

FELLOWSHIP OF POSTGRADUATE MEDICINE

60 Portland Place, London, W.I 